\title{
Getting down to details
}

\section{MicroRNAs that tweak gene expression, single nucleotide polymorphisms in population genetics, and individual genome sequencing: Caitlin Smith takes a look at three fast-moving areas in genomics.}

Over the past few years, genomics researchers have been getting to grips with a 'new' genome element - microRNA (miRNA). Although a small number of miRNAs have been familiar to developmental biologists for years, a plethora of miRNAs has recently been discovered in animal and plant genomes. More than 200 miRNAs have been identified in mammalian genomes, but their functions mostly remain a mystery. Silencing gene expression in a similar way to small interfering RNAs (see Nature 431, 350; 2004), mammalian miRNAs are implicated in the control of cell and tissue differentiation, apoptosis, insulin secretion, fat metabolism and cancer.

"We are now aware that there is substantially more transcription from human chromosomes than can be accounted for by the current predictions of human genes," says Frank Slack at Yale University, New Haven, Connecticut. Slack is studying the apparent involvement of the miRNA let-7 in lung cancer and the implications of its ability to suppress translation of the oncogene RAS. "Many miRNAs are mapping to disease loci where previously a gene was not found," he says.

miRNA research is a typical microcosm of

the variety of disciplines and techniques that are required to make sense of the genome - computational biology, bioinformatics and comparative genomics to predict candidate miRNAs, followed by classic 'wet biology' to validate the candidates and study their expression and function. And as more labs are gearing up to study miRNAs, commercial products tailored to help them are coming onto the market.

The technical problems of detecting miRNAs in total cellular RNA stem from their small size and often low abundance. Produced

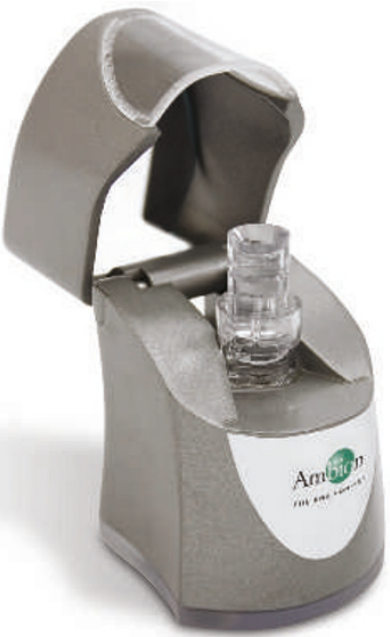

Micro solution: Ambion's flashPage isolates small RNAs. from a larger precursor molecule, mature miRNAs are RNA hairpins of 17-23 nucleotides, which bind to complementary sequences in their target messenger RNAs (mRNAs) and prevent translation. The general techniques for detecting and isolating miRNAs from cellular RNA are those used for other small RNAs. A first step could be spincolumn fractionation of RNA to remove larger RNAs, using columns such as the Amicon YM-100 from Millipore of Bedford, Massachusetts, which will remove RNAs of more than 75 bases, or the PureLink miRNA isolation kit from Invitrogen of Carlsbad, California, with a 200nucleotide limit. Qiagen of Valencia, California, has a small RNA protocol for their widely used RNeasy system, which will remove RNAs of more than 200 bases from total cellular RNA. To get even closer to mature miRNA length, RNA specialists Ambion of Austin, Texas, sells a flashPage, a gel-based fractionation machine for the rapid isolation of small nucleic acids of around 40 bases.

After initial preparation, specific miRNAs

\section{BIG TASKS FOR SMALL MOLECULES}

Having helped to identify many miRNAs, Christopher Burge and his colleagues at the Massachusetts Institute of Technology are one of many teams now tackling an even bigger job to find out which genes are regulated by the known miRNAs, and how they fit into physiological pathways.

Finding targets begins computationally, using the TargetScan algorithms developed by Benjamin Lewis working with Burge and with David Bartel at the Whitehead Institute for Biomedical Research in Cambridge, Massachusetts. These algorithms "rely on evolutionary conservation of segments complementary to the microRNA 'seed' region in the 3 ' untranslated regions of orthologous genes from multiple vertebrate organisms", says Burge. The seed region, six or seven bases at the 5 ' end of the miRNA, is thought to be key to

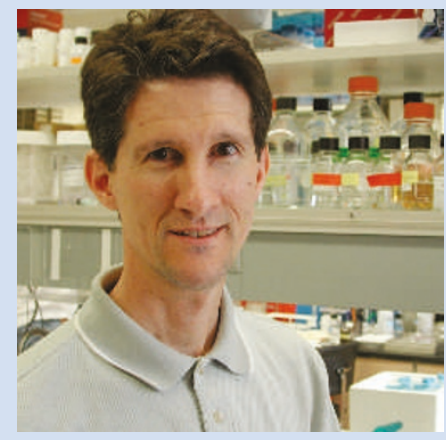

James Carrington: taking a systems look at miRNA.

specifying which genes an miRNA will regulate. Targets have been verified in Bartel's lab using a dual luciferase reporter system, which measures the effect of predicted miRNA interaction sites on protein production in cultured human cells. In a computational analysis published earlier this year, Lewis, Burge and Bartel estimated that more than a third of our genes might be regulated by miRNAs.
The task will be complicated by the fact that an miRNA may regulate as many as $\mathbf{2 0 0}$ genes, according to a computational study by Nikolaus Rajewsky and his colleagues at New York University and Rockefeller University, using their PicTar algorithm to identify miRNA targets. Other software for miRNA target prediction includes miRANDA from Anton Enright and his colleagues at the Memorial Sloan-Kettering Cancer Center in New York and DIANA-microT from Artemis Hatzigorgeou and Axel Bernal at the University of Pennsylvania, Philadelphia.

Frank Slack's team at Yale University uses in situ hybridization, northern blots and fluorescent protein fusions to find when and where miRNAs and their targets are expressed. "We use genetics and RNA interference to reduce the expression of potential targets to see if we suppress the effects of a mutation in the corresponding miRNA, and use reporter gene assays to test if the miRNAcomplementary sites function in gene regulation," he says.

"The classic tools of developmental biology and physiology are needed to correlate miRNA expression and targeting to biological function," agrees James Carrington at Oregon State University, Corvallis, who is looking at pathways regulated by miRNAs in Arabidopsis. "miRNA sensors involving miRNA target sites within gene constructs expressing a fluorescent protein are quite useful in understanding spatial and temporal miRNA expression and activity patterns," he says. But to address the question of how miRNAs integrate with cellular pathways, "the more quantitative approaches using the tools of systems biology and computational analysis are the trend in this lab", he says. 